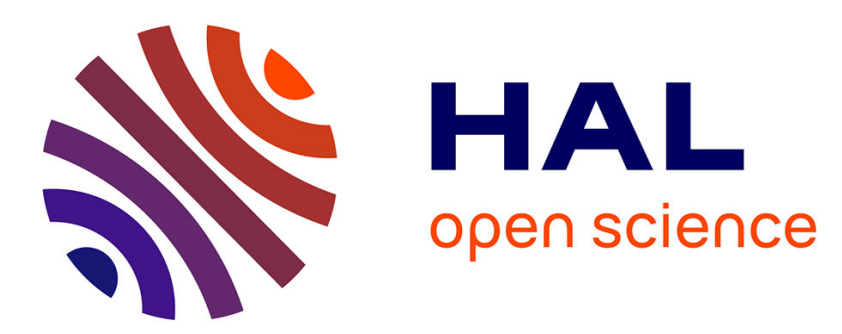

\title{
Vienne, contraintes hydrologiques et aménagements des rives du Rhône: de la komè allobroge à la ville du Haut-Empire
}

\author{
Anne Le Bot-Helly, Benoît Helly
}

\section{To cite this version:}

Anne Le Bot-Helly, Benoît Helly. Vienne, contraintes hydrologiques et aménagements des rives du Rhône: de la komè allobroge à la ville du Haut-Empire. Gallia - Archéologie de la France antique, 1999, Le Rhône romain, 56, pp.71-79. 10.3406/galia.1999.3244 . hal-01913001

\author{
HAL Id: hal-01913001 \\ https://hal.science/hal-01913001
}

Submitted on 16 Jan 2020

HAL is a multi-disciplinary open access archive for the deposit and dissemination of scientific research documents, whether they are published or not. The documents may come from teaching and research institutions in France or abroad, or from public or private research centers.
L'archive ouverte pluridisciplinaire HAL, est destinée au dépôt et à la diffusion de documents scientifiques de niveau recherche, publiés ou non, émanant des établissements d'enseignement et de recherche français ou étrangers, des laboratoires publics ou privés.

\section{(ㅇ)(1) $\$$}

Distributed under a Creative Commons Attribution - NonCommercial - NoDerivatives $\mid 4.0$ 


\title{
VIENNE, CONTRAINTES HYDROLOGIQUES ET AMÉNAGEMENTS DES RIVES DU RHÔNE
}

\author{
De la komè allobroge à la ville du Haut-Empire
}

\author{
Anne Le Bot-Hel.Ly avec la collaboration de Benoît Het.LY
}

Mots-clés. Rhône, Allobroges, Âge du Fer, Antiquité, Vienne, Saint-Romain-en-Gal, Sainte-Colombe, département de l'Isère, département du Rhône, remblais, urbanisme, crues.

Key-words. Rhône, Allobroges, Iron Age, Antiquity, Vienne, Saint-Romain-en-Gal, Sainte-Colombe, department of Isère, department of Rhône, embankments, urbanism, floods.

Résumé. À partir de la fin du $V^{e}$ s. avani J.-C., une agglomération s'est constituée sur un promonloire surplombant la rive gauche du Rhône et son confluent avec la Gère. Cette capitale des Gaulois allobroges devenue cheflieu d'une très vaste cité romaine dut sa pérennité et son développement à sa situation sur le Rhône, au carrefour de plusieurs vallées. L'étude du terrain naturel menée systématiquemenl depuis une quinzaine d'années à l'occasion des fouilles archéologiques montre que l'agglomération gauloise, à l'origine perchée sur son promontoire bordé par les cours d'eau, n'a pu devenir une des villes les plus vastes de la Gaule qu'à la suite d'une modification progressive du régime du Rhône initiée dès le II s. avant J.-C. et sensible jusqu'au tournant de notre ère. À cette époque, le site présentait sur chaque rive du fleuve deux vastes plaines alors hors de porlée des inondations. Les premières constructions observées sur ces terres basses montrent que, tout en restant proche du niveau du terrain naturel, on prit quelques précautions pour lutter contre l'humidité (place Saint-Pierre : sol sur vide sanitaire en amphore, place Camille-Jouffray : épandages de céramiques, Saint-Romain-en-Gal : cloisons de bois et de terre sur des murs bahuts en maçonnerie). Entre les années 20 et 50 après J.-C., des mesures d'une autre ampleur furent prises : les quartiers de la plaine sur les deux rives furent rapidement exhaussés de 1,50 m à près de $3 \mathrm{~m}$ selon les secleurs. Plus qu'une précaution définitive d'urbaniste, ces aménagements giganlesques au regard des surfaces concernées pourraient être une réponse à un relour temporaire des crues dont on a retrouvé quelques témoignages dalables des années 30-40 au sud de la ville.

\begin{abstract}
Since the end of the $5^{\text {th }}$ century $B C$, a town has been established on a hill overlooking the eastern bank of the Rhône and its confluence with the Gère. Main town of the Allobroges, it became a large Roman civitas capital and long continuily and development are due to its position in the Rhône valley' at the junction of several valleys. Study of geology carried out systematically since fifteen years, during excavations, reveals that the native settlement, originally perched on a promontory surrounded by streams, became one of the largest towns in Gaul, after progressive modification of the river system that starled as soon as the $2^{\text {nd }}$ century BC, and noticeable up to the turn of Christian era. At that time, the settlement was lying on two flat-lands on both banks of the Rhône out of reach of floods. The first buildings noticed on the low-lands show that even being near the natural ground level, some precautions were taken against dampness (place Saint-Pierre : underfloor space made of amphorae ; place Camille-Jouffray : spreads of pottery sherds; Saint-Romain-en-Gal : wooden and earth internal divisions on masoned "murs bahuls"). Between 20 and $50 \mathrm{AD}$, extensive measures were taken : the lower districts on both banks were quickly raised, from $1,50 \mathrm{~m}$ high up to $3 \mathrm{~m}$, varying with the areas. These huge equipments in relation to the areas concerned are not really the achievement of cautious town-planners but could be an answer to a temporary return of floods of which evidence dated of the years 30-40, was yielded south of town.
\end{abstract}




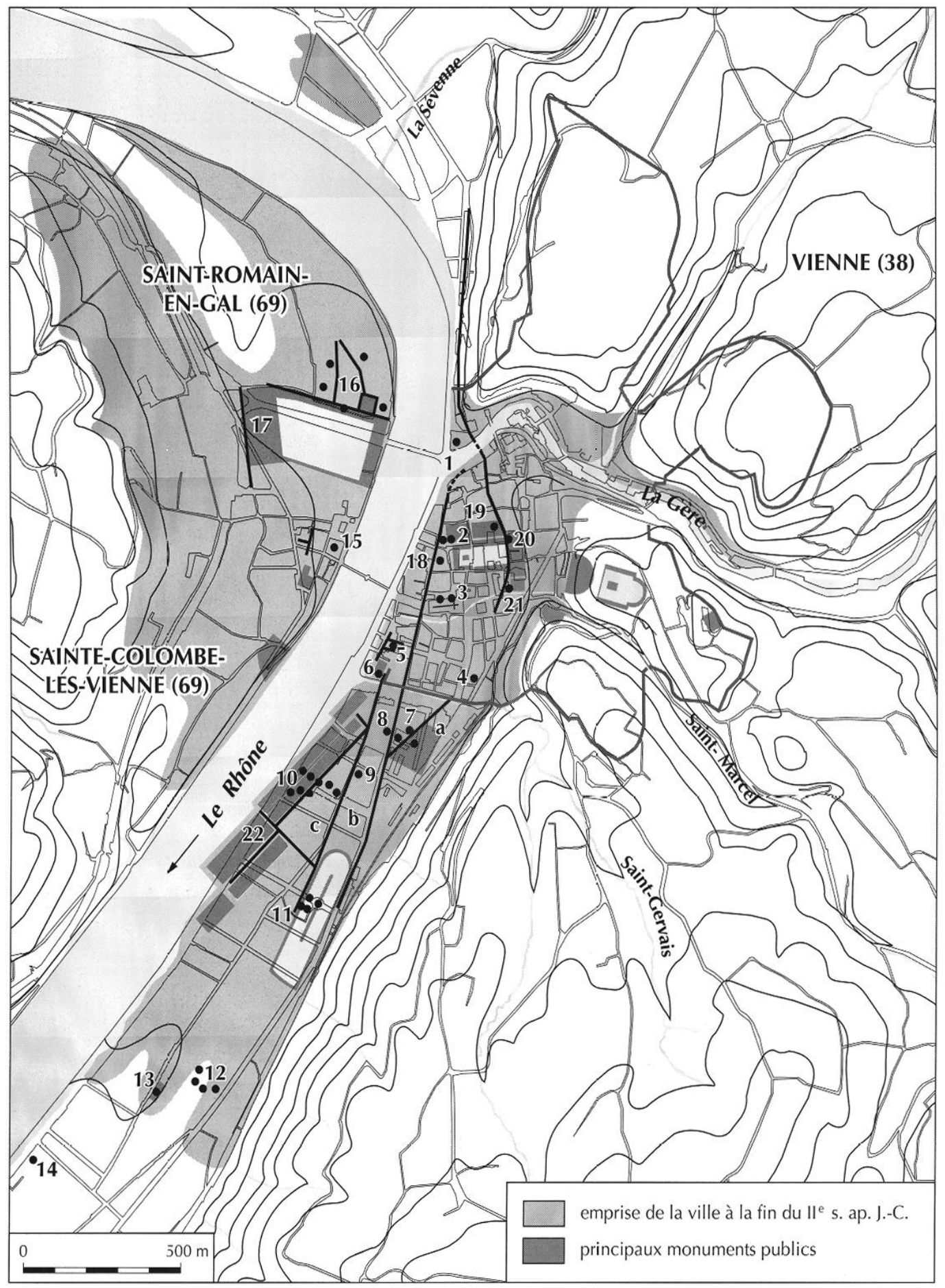

Fig. 28 - Situation des points d'observation principaux : a, voie du Fanum ; b, voie du Cirque; $c$, voie Médiane (DAO C. Marcellin, $A F A N)$.

Vienne (Isère) : 1, Notre-Dame-d'Outre-Gère ; 2, Palais de Justice; 3, Saint-Maurice; 4, Banque de France; 5, place Saint-Pierre et drain CNR; 6, cours Brillier ( $\left.n^{\circ} 16\right) ; 7$, place Camille-Jouffray; 8, place des Allobroges; 9, rue Laurent-Florentin; 10, ZAC des Nymphéas; 11, cirque ; 12, terrain Arnaud ; 13, terrain Devun ; 14, rue Jean-Moulin ; 18, 61 rue de Bourgogne; 19, Hôtel de Ville; 20, rue des Orfêres (prolongement sud de la rue Marchande); 21, jardins de Cybèle; 22, quai Riondet ( $\left.n^{\circ} 35\right)$.

Sainte-Colombe (Rhône) : 15, rue Paul-Doumer.

Saint-Romain-en-Gal (Rhône) : 16, site archéologique ; 17, Palais du Miroir. 
En 1989, une première interprétation de l'évolution de la plaine alluviale du Rhône et de ses relations avec l'histoire de la topographie urbaine, de l'Âge du Fer à la fin de l'Antiquité, a été proposée à la suite d'une étude interdisciplinaire portant sur l'ensemble des sondages archéologiques réalisés sur les deux rives (Bravard et al., 1990). Depuis cette date, quelques informations complémentaires recueillies dans le centre ancien tendent à confirmer les hypothèses présentées alors ${ }^{42}$ (fig. 28).

L'évolution de la plaine alluviale n'a été qu'un des facteurs déterminants dans le développement de la ville. Il s'y est ajouté au cours de la première moitié du $I^{\mathrm{er}} \mathrm{s}$. après J.-C. une forte volonté politique : celle de créer dans cette plaine, malgré le Rhône, une grande partie des quartiers neufs de la ville, sur des terrains artificiels épais de 1,5 à $3 \mathrm{~m}$. Il en est ainsi du « quartier sud » sur la rive gauche et du « quartier de Saint-Romain-en-Gal » sur la rive droite (Desbat et al., 1994 ; Brissaud, Prisset, 1996) (fig. 31).

\section{DES ORIGINES À LA CONQUÊTE}

Le centre historique de Vienne est aujourd'hui établi sur 6 à $9 \mathrm{~m}$ de dépôts archéologiques. Trois sondages (fig. $28 ; n^{\circ} 21$ cf. Pelletier, $1966 ; \mathrm{n}^{\circ} 19$ cf. Le Bot-Helly, $1992 ; n^{\circ} 20$ cf. Le Bot-Helly, 1995b) montrent que c'est sur un promontoire dominant d'une quinzaine de mètres environ le confluent de la Gère et du Rhône que s'installe vers la fin du $V^{c} s$. ou au début du IV $\mathrm{I}^{\mathrm{e}} \mathrm{s}$. avant J.-C. une première agglomération (?). À cette époque, le Rhône passe immédiatement au pied de ce promontoire (fig. 28, $n^{\circ}$ 2) et recouvre en partie le secteur de la place Camille-jouffray (fig. 28, ${ }^{\circ}$ 7), (Bravard et al., 1986; Baratte et al., 1990). Sur l'autre rive, alors que le fleuve balaye la plaine de Saint-Romain-en-Gal, le rebord de la terrasse würmienne de Sainte-Colombe (155-153 m NGF) est hors de portée des eaux les plus hautes (fig. 28, $\mathrm{n}^{\circ} 15$ et fig. 29).

Le promontoire est limité au nord-est par une barre rocheuse qui surplombe la vallée de la Gère (fig. 29). Du

42. Nous ne revenons pas ici sur les données géomorphologiques exposées en 1990. L.es détails stratigraphiques seront exposés dans l'Atlas archéologique, en cours de préparation. côté du Rhône, il est bordé par une autre barre rocheuse qui se trouve à la cote 163,7 m NGF (fig. 29). Dominé à l'est par la colline de Pipet (252 m NGF), il est limité au sud par le vallon du ruisseau Saint-Marcel. Les sondages (fig. 28, $\mathrm{n}^{\text {os }} 19,20,21$ ) montrent qu'il est traversé par un ensellement nord-sud dont le niveau varie entre $158,5 \mathrm{~m}$ NGF (fig. 28, $\mathrm{n}^{\circ}$ 20) et 161,5 m NGF (fig. 28, $\mathrm{n}^{\circ} 21$ ). C'est dans cette légère dépression qu'ont été mis en évidence les niveaux d'occupation remontant à la fin du $\mathrm{V}^{\mathrm{e}} \mathrm{s}$. ou au début du IV $\mathrm{I}$ s. avant J.-C. Il est possible que ce premier habitat se soit installé le long d'un chemin qui, en empruntant l'ensellement, permettait de franchir le promontoire en évitant la berge abrupte du Rhône (fig. 29). Dans la direction du nord, ce chemin devait suivre un tracé proche de l'actuelle rue Marchande. En effet, à l'est de cette rue, la berge sud de la Gère domine brutalement le cours d'eau (de 10 à $20 \mathrm{~m}$ ) et ne s'abaisse que $5 \mathrm{~km}$ plus à l'est.

Dans la plaine alluviale, les premiers témoins d'un habitat datent du deuxième quart du $\mathrm{II}^{\mathrm{e}} \mathrm{s}$. avant J.-C. (Kroichvili, 1993). Installée au pied même du promontoire (fig. $28, \mathrm{n}^{\circ}$ 2), sur des éboulis rocheux, à la cote 149,5 m NGF environ, une première maison est détruite par une inondation. Un second habitat s'installe "définitivcment " au même emplacement au milieu du $\mathrm{II}^{\mathrm{c}} \mathrm{s}$. avant J.-C., à la cote $150 \mathrm{~m}$ NGF environ. Il semble bien qu'à partir de cette période le Rhône ait changé de régime : sa berge s'est décalée vers l'ouest et ses eaux les plus hautes n'atteignent plus la cote $150 \mathrm{~m}$ NGF à partir du milieu du II's.

Un peu à l'écart de cette agglomération (?), le sommet de la colline de Sainte-Blandine est occupé à partir du premier quart du $\mathrm{II}^{\mathrm{c}} \mathrm{s}$. avant J.-C. La nature du mobilier recueilli apparente le site à un sanctuaire (Chapotat, 1970 ; Perrin, 1990).

\section{DE LA CONQUÊTE À LA PÉRIODE AUGUSTÉENNE}

En 121 avant J.-C., les Allobroges sont soumis. Strabon (IV, 1, 11) précisera 140 ans plus tard que ces Allobroges vivent dans des bourgades (komè), sauf les plus illustres d'entre eux qui, installés à Vienne, ont fait de celle-ci, qui n'était précédemment qu'une komè portant le titre de metropolis, une ville (polis) bien équipée. Mais à quelle 
période précisément Strabon fait-il allusion ? Est-ce que cette transformation est liée à la conquête? Nous connaissons très peu de chose concernant l'évolution de l'agglomération entre la fin du II ${ }^{\mathrm{e}} \mathrm{s}$. et le milieu du ${ }^{\text {er }} s$. avant J.-C. Un monument public (?) occupe le bord occidental du promontoire, dès les années 80 avant J.-C. ; au pied du promontoire (fig. $28, n^{\circ} 2$ ), après plusieurs reconstructions à la cote $150 \mathrm{~m} \mathrm{NGF}$, une maison est

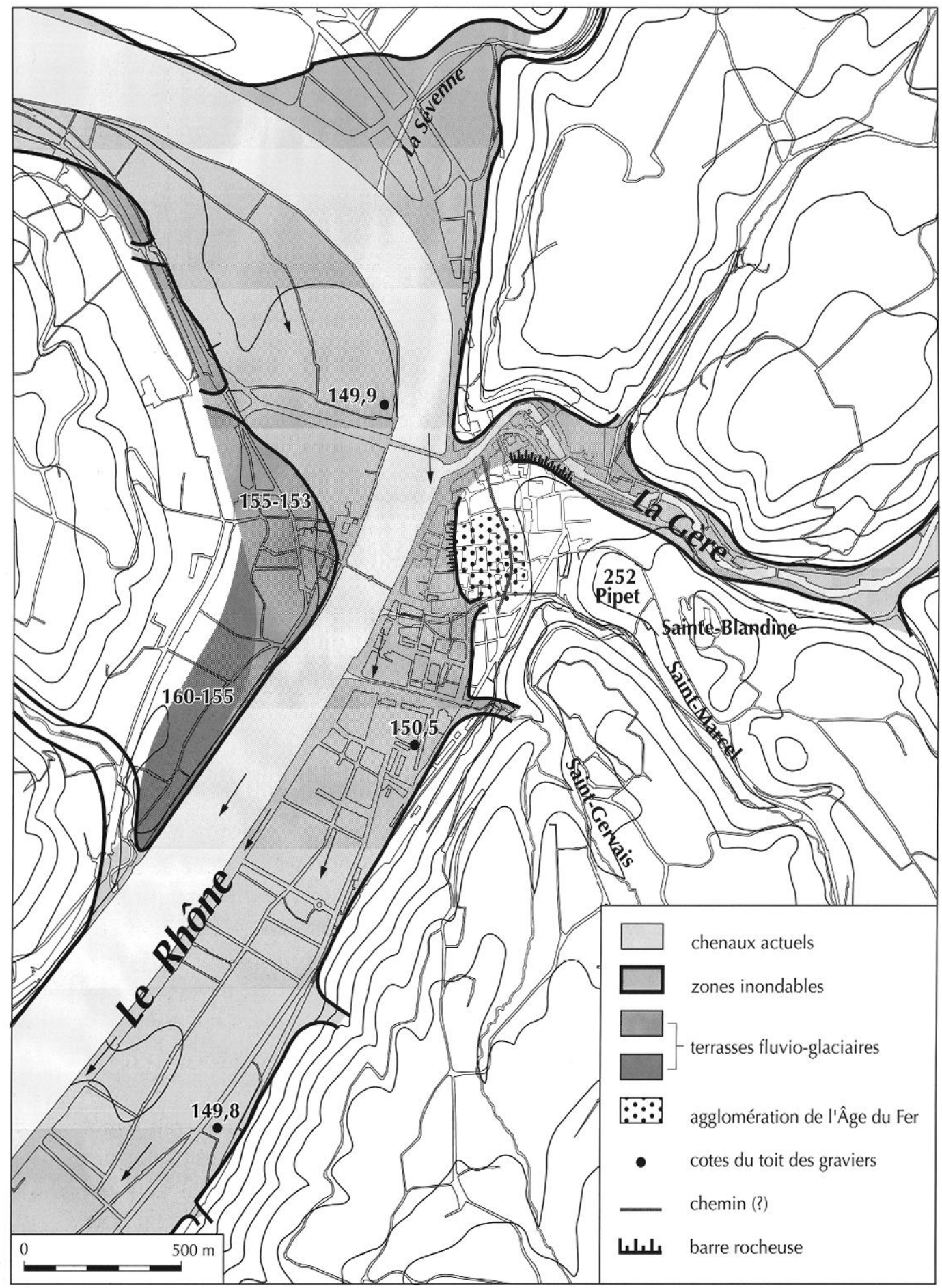

Fig. 29 - Vienne du V's. avant J.-C. à la fin du III s. avant J.-C. (DAO C. Marcellin, AFAN). 
édifiée après les années 80 avant J.-C., à la cote 150,5 m NGF (Kroichvili, 1993). La fixation définitive de l'habitat dans la plaine alluviale depuis 150 avant J.-C. suggère qu'un chemin longeait désormais la berge du Rhône pour rallier plus au nord l'embouchure de la Gère (fig. 30).

$\mathrm{Au}$ début de la conquête, il semble que le Rhône passe encore sous l'emprise de la cathédrale SaintMaurice (fig. 28, $n^{\circ} 3$ ), si l'on en croit une coupe stratigraphique levée vers $1861^{43}$. Le toit des graviers dit « graviers solides » varie entre 145,3 m NGF à l'est (puits IX) et $146,45 \mathrm{~m}$ NGF à l'ouest (puits VIII). S'il y avait un chemin de berge (fig. 30), il devait passer alors à l'est du chevet de la cathédrale et se diriger vers le site de la Banque de France (fig. 28, n ${ }^{\circ} 4$ ), où une terrasse recouverte de colluvions se situait à cette période à la cote 155,5 m NGF environ (Canal, 1982). Plus au sud, au-delà du ruisseau Saint-Gervais, le point le plus haut $(152 \mathrm{~m}$ NGF) de la terrasse de la place Camille-Jouffray (fig. 28, $\mathrm{n}^{\circ} 7$ ) ne semble avoir été occupé par un fanum qu'à partir des années 30-20 avant J.-C. ; à une centaine de mètres à l'ouest, une ou deux décennies avant, le bord de la berge du Rhône qui se trouvait alors sous le tracé de l'actuel cours de Verdun (place des Allobroges) servait d'aire d'épandage (fig. $28, \mathrm{n}^{\circ} 8$ et fig. 30 ).

Au cours du I ${ }^{\text {er }}$ s. avant J.-C., le retrait du lit du Rhône paraît avoir été suffisamment important, pour que l'on s'installe sporadiquement (?) plus à l'ouest à des niveaux assez bas, sur les derniers dépôts d'inondations comme le montrent les coupes réalisées au pied de la cathédrale Saint-Maurice (fig. 28, $n^{\circ} 3$ ). Du côté est (puits XI), les premiers niveaux d'occupation « amphores cassées, poteries, terre noirâtre » se situent à la cote 147,6 m NGF ; du côté ouest (puits IX), sur des dépôts successifs « sable, marnes graveleuses, marnes fines " qui témoignent du retrait progressif du Rhône, du " bois " (un aménagement de berge ?) est signalé entre les cotes 146,7 m NGF et $149 \mathrm{~m} \mathrm{NGF}$; un niveau d'occupation " couche noire et amphores " est mentionné au-dessus de cet aménagement à la cote 149 m NGF.

$\mathrm{Au}$ cours de la période augustéenne, sur le bord occidental de la place Saint-Pierre (fig. $28, \mathrm{n}^{\circ}$ ), un

43. Anonyme, Église Saint-Maurice, plan d'attachement des reprises en sous auvre du collatéral nord : élévation des puits nord, (travaux exécutés en 18601861), Monuments Historiques, Département de l'Isère, Ville de Vienne, plan original dressé par H. Quenin (?), mis au net le 15 juillet $1910,1 / 50$, document Monuments historiques. sol construit sur un vide sanitaire constitué d'amphores renversées (Dressel 9) est établi à la cote 148,25 m NGF sur les limons du Rhône. Plus au sud, 16 rue Laurent-Florentin (fig. 28, $\mathrm{n}^{\circ}$ 9), les restes d'une petite installation métallurgique (?) ont été observés à la cote 148 m NGF. Directement installée sur des dépôts de débordement du Rhône datables du $I^{\text {er }} \mathrm{s}$. avant J.-C., cette installation appartient au début de l'époque augustéenne. C'est à la même époque que l'on construit, plus à l'est, deux rues, la voie du Cirque (fig. 28, b) établie sur des remblais à la cote $151,3 \mathrm{~m} \mathrm{NGF}$ et la voie du Fanum (fig. 28, a) installée directement sur la terrasse de l'Âge du Fer à la cote151,8 m NGF, sur le site de la place Camille-Jouffray (fig. 28, $n^{\circ} 7$ ). La voie nord-sud (la voie du Cirque) se trouve dans le prolongement exact d'une voie reconnue au $\mathrm{XIX}^{\mathrm{e}}$ s., au nord, sous les actuelles rue Boson et rue de Bourgogne. Cet axe qui passe au pied du promontoire, peut-être à l'emplacement du chemin dont nous supposons l'existence à partir du II $\mathrm{e}$ s. avant J.-C., limite alors la ville à l'ouest. À cette époque, l'établissement d'une voie carrossable au pied du promontoire, rendu possible grâce au retrait du fleuve, devenait indispensable : elle évitait le promontoire où, à l'époque augustéenne, on construisait le forum sur unc vastc plate-forme, à l'emplacement de l'ensellement originel remblayé pour l'occasion jusqu'à la cote $161 \mathrm{~m}$ NGF.

Le long du Rhône, cette rue devenait désormais un axe commercial essentiel. Nous supposons qu'un premier quartier augustéen occupait alors tout l'espace situé dans la plaine alluviale à l'est de cette voie. Les points d'observation sont très rares dans ce secteur. Un entrepôt de céramiques (fig. $28, n^{\circ} 18$ ) est établi à la cote 153,8 m NGF sur un mur de soutènement renforcé à l'arrière par des absides (Le Bot-Helly, 1991 ; Godard, 1992).

Sur la rive droite du Rhône, la plus ancienne occupation connue a été observée à Sainte-Colombe (fig. 28, $n^{\circ} 15$ ), où un dépotoir du milieu du $\mathrm{I}^{\mathrm{er}} \mathrm{s}$. avant J.-C., très partiellement fouillé au bord de la terrasse würmienne, à la cote 153 m NGF, pourrait témoigner de la proximité d'un habitat (Helly, 1994). La plaine alluviale de SaintRomain-en-Gal (fig. 28, $\mathrm{n}^{\circ} 16$ ), progressivement libérée par le fleuve à partir du $\mathrm{II}^{\mathrm{e}} \mathrm{s}$. avant J.-C., est occupée vers 40 avant J.-C. Un espace agricole (?) est organisé autour de deux chemins empierrés bordés de fossés. Une borne prouve l'existence d'une division parcellaire. Le premier 
quartier augustéen établi sur les dépôts de débordement du fleuve se caractérise par une occupation diffuse associant des ateliers et quelques habitats situés en bordure de rues. La maison à la Citerne est créée vers 20 avant
J.-C. et la première rue est-ouest (voie I) qui la limite au sud quelques années avant, à la cote 149,5 m NGF, alors que la voie nord-sud (voie II) n'est créée que vers 10-15 après J.-C. à $0,7 \mathrm{~m}$ plus haut que la voie $\mathrm{I}$.

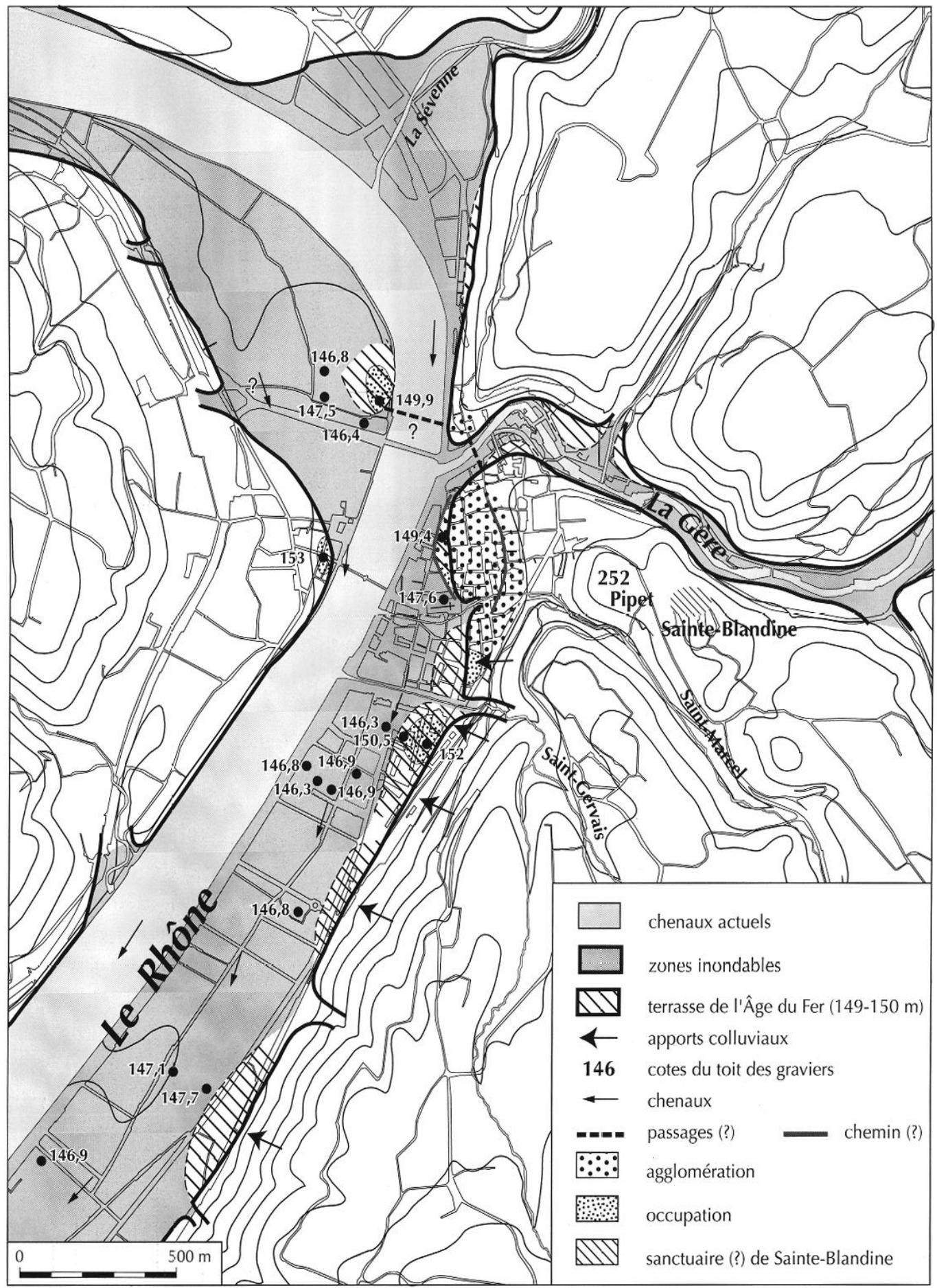

Fig. 30 - Vienne du II s. avant J.-C. jusqu'aux années 40-30 avant J.-C. (DAO C. Marcellin, AFAN). 


\section{LA PÉRIODE TIBÈRE-CLAUDE}

Au tout début du Irr s. après J.-C., les berges libérées par le Rhône (aucun témoin de débordement du fleuve n'a été retrouvé pour cette période) devaient, au moins dans la mémoire collective, rester potentiellement inondables. À partir des années 15-20 après J.-C., on constate la mise en oeuvre d'aménagements particulièrement importants pour exhausser la plaine alluviale et créer de nouveaux quartiers (fig. 31).

En rive gauche, à l'ouest de la voie du Cirque (fig. 28, b), toutes les observations archéologiques concordent. Les nouveaux bâtiments sont établis sur des fondations, construites en partie en élévation, qui sont remblayées sur 1,50 à $3 \mathrm{~m}$ de hauteur par des matériaux fins d'origine fluviatile entre lesquels on intercale souvent des déchets de carrière qui devaient avoir des qualités drainantes.

Au début du règne de Tibère, un quartier est établi plus à l'ouest, entre la voie du Cirque (fig. 28, b) et la voie Médiane (fig. 28, c). Une grande maison est construite place Saint-Pierre (fig. 28, $n^{\circ} 5$ ) à la cote 151,1 m NGF (Tourrenc, 1975; Lancha, 1981 ; Barbet, 1982) dans un îlot limité à l'ouest par une voie nordest/sud-ouest qui suivait peut-être un coude du Rhônc. $\grave{\Lambda}$ la même époque, on édifia, à une centaine de mètres au sud, l'angle sud-ouest de l'enceinte (Le Bot-Helly, 1991) qu'une voie borde le long du Rhône (fig. 28, $n^{\circ} 6$ ). Cette voie Médiane (fig. 28, c) se prolongeait au sud sous l'actuelle rue du Onze-Novembre. Le sondage réalisé rue Laurent-Florentin (fig. 28, $\mathrm{n}^{\circ}$ 9) a montré qu'elle limitait dans ce secteur un îlot établi à la cote 149,8 m NGF. Plus au sud en revanche, à la hauteur de la " pyramide " du cirque du II's., le secteur situé à l'est de l'axe de la voie Médiane reste à la cote 148,7 m NGF jusqu'aux années 60-70 après J.-C. Quelques niveaux d'occupation ont été repérés sur des dépôts de débordement situés à $148,1 \mathrm{~m}$ NGF et datables du I ${ }^{\text {er }}$ s. avant J.-C. (fig. 28, $n^{\circ} 11$ ).

Enfin, vers 30-40 après J.-C., est créé encore, à l'ouest de la voie Médiane, un dernier quartier le long du fleuve. Les très nombreux sondages réalisés sous l'emprise de la ZAC des Nymphéas (fig. 28, $\mathrm{n}^{\circ} 10$ ) ont montré que les crues de débordement du Rhône touchaient encore cette zone au milieu du $\mathrm{I}^{\mathrm{cr}} \mathrm{s}$. avant J.-C. Dans la dernière décennie du siècle, le ruisseau Saint-Gervais incise les dépôts du Rhône. Son cône de déjection portera les niveaux à la cote 148,8 m NGF. Le ruisseau ayant été canalisé au nord (fig. 28, $n^{\circ} 6$ ) vers les années 15-20 après J.C. (Le Bot-Helly, 1995a), vers 30-40 après J.-C. on établit sur cette zone à la cote 150,8 m NGF, en remblayant sur 2 à $3 \mathrm{~m}$ de hauteur, les plus grands horrea publics connus à ce jour dans les provinces occidentales. Pendant leur construction, une crue vient ronger les berges nouvellement établies, mais les eaux du Rhône ne touchent pas le sommet des remblais. Les dépôts de sédiments fins ne dépassent pas à cette occasion la cote 148,2 m NGF (Bravard et al., 1990).

À la même période, en rive droite, le quartier de Saint-Romain-en-Gal est aussi réaménagé et exhaussé (fig. 28, $\mathrm{n}^{\circ} 16$ ). Vers 50 après J.-C., la maison aux Pierres Dorées, la maison au Grand Péristyle, les Grands Entrepôts sont construits sur des remblais qui portent les sols au minimum à la cote $150,5 \mathrm{~m}$ NGF et plus souvent à la cote $151 \mathrm{~m} \mathrm{NGF}$ en moyenne. La maison au Vivier évolue de manière particulière : le bâtiment à l'est est exhaussé jusqu'à la cote $152 \mathrm{~m}$ NGF, pendant que le jardin à l'ouest reste au niveau du terrain naturel initial : 149,6 m NGF (Savay-Guerraz et al., 1992). Entre les années 50 et 70 après J.-C., le complexe thermal du Palais du Miroir est construit (Savay-Guerraz, Prisset, 1992). Tous les bâtiments édifiés autour de l'esplanade centrale sont fortement cxhaussćs sur des remblais : lc sol du grand portique nord est porté à 152,3 m NGF et celui du bâtiment qui longe le Rhône à la cote $152 \mathrm{~m}$ NGF au moins. Les sols du Palais du Miroir à l'ouest se trouvent à la cote $154 \mathrm{~m} \mathrm{NGF}$ (fig. $28, \mathrm{n}^{\circ}$ 17). Le bâtiment s'appuie en partie sur le rebord d'une terrasse würmienne; du côté de la plaine alluviale, il se développe vers l'est sur un système de voûtes parallèles. L'esplanade centrale établie à la cote 150,1 m NGF n'est exhaussée que de $2 \mathrm{~m}$. Jouant sur les niveaux, cet aménagement relève d'un projet architectural. La position basse de l'esplanade entre les trois constructions évitait des travaux coûteux et en outre permettait à l'eau de s'étaler en cas de crue.

À la fin de la période julio-claudienne, les quartiers construits dans la plaine alluviale, sur les deux rives, se trouvent au minimum à la cote $150,8 \mathrm{~m} \mathrm{NGF}$ (sauf l'esplanade centrale du complexe du Palais du Miroir). Cinquante hectares environ ont été ainsi exhaussés de $2 \mathrm{~m}$ en moyenne ( 1 million de $\mathrm{m}^{3}$ !) en une quarantaine d'années. D'après la cote de certains égouts, le niveau moyen du Rhône peut être évalué à la cote $146 \mathrm{~m}$ NGF. 


\section{LA FIN DU I ${ }^{\text {er }}$ S. ET LE II ${ }^{\mathrm{e}} \mathrm{S}$.}

À la fin du I ${ }^{\text {er }}$ s. après J.-C., au sud-est des grands horrea de la rive gauche (fig. $28, \mathrm{n}^{\circ} 10$ ), la construction d'un premier cirque donne lieu à l'un des derniers aménagements importants de la plaine sud. La piste est établie à la cote $150 \mathrm{~m}$ NGF environ, alors que les niveaux de circulation extérieurs de l'édifice sont plus hauts de $0,7 \mathrm{~m}$,

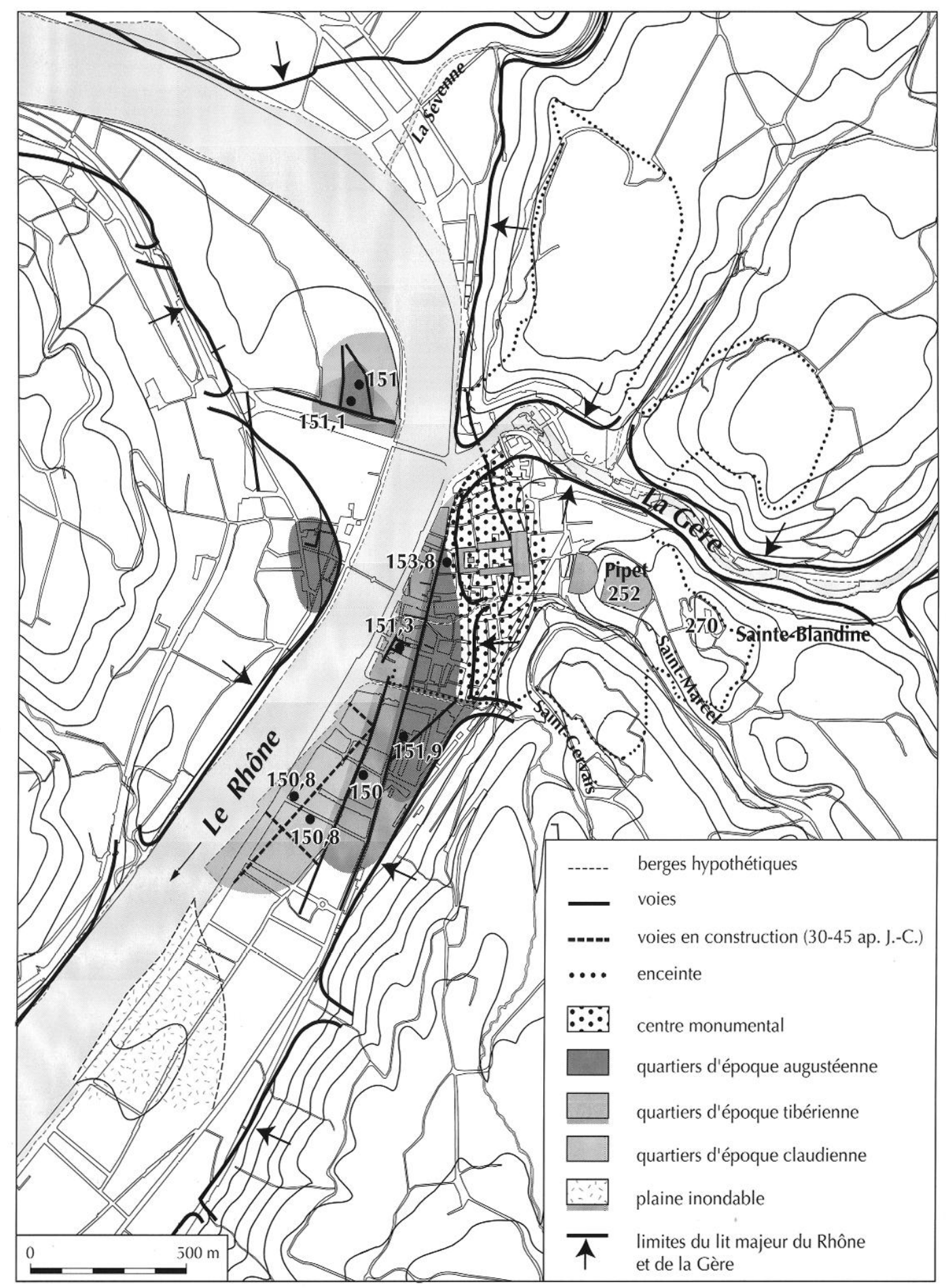

Fig. 31 - Vienne au cours du deuxième quart du Irr s. après J.-C. (DAO C. Marcellin, AFAN). 
afin de se raccorder au niveau général du quartier. Ce système de construction est celui adopté pour le complexe du Palais du Miroir. À la fin du II ${ }^{\mathrm{e}}$ s., le cirque est rebâti : l'ensemble des sols intérieurs et extérieurs sont alors établis à la même cote, $151 \mathrm{~m}$ NGF en moyenne (Le Bot-Helly, à paraître). La limite de la ville se situe au sud de ce monument où les terrains non remblayés subiront les crues du Rhône au moins jusqu'à la moitié du $\mathrm{II}^{\mathrm{c}} \mathrm{s}$. Sur un niveau de graviers situé à 147,4 m NGF, des dépôts fins de débordement du Rhône sont attestés à partir du milieu du Irr $\mathrm{s}$. après J.-C. et se succèdent jusqu'à la cote $149 \mathrm{~m}$ NGF (fig. 28, $\mathrm{n}^{\circ}$ 12). Plus à l'ouest (fig. 28, $\left.\mathrm{n}^{\circ} 13\right)$, au II ${ }^{\mathrm{c}} \mathrm{s}$., un entrepôt est bâti sur des niveaux de débordement situés eux aussi à la cote 149 m NGF. Un bras mort borde l'est de ces entrcpôts construits le long du prolongement de la voie Médiane.

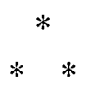

En un siècle et demi, la komè que les Allobroges avaient choisie comme capitale au confluent du Rhône et de la Gère devient une des plus grandes villes de la Gaule (300 ha dont près de 200 ha bâtis). Ce développement s'est effectué autour d'un point élevé qui, à l'origine, offrait au mieux une surface d'une quinzaine d'hectares. Vienne avait été fondćc sur un sitc qui répondait bien aux besoins d'une population de la Tène : un point haut protégé par la colline de Pipet, des éperons rocheux et des cours d'eau, et surtout situé sur un nœud de communications essentiel qui permettait de relier la vallée du Rhône et les routes alpines par les vallées de la Gère et de Saint-Marcel. De plus, à une dizaine de kilomètres au nord, la vallée du Gier ouvrait, à travers le territoire des Ségusiaves, sur la vallée de la Loire.

Lorsqu'à la fin du I ${ }^{\mathrm{cr}}$ s. avant J.-C., Auguste réorganisa la Province et que Vienne fut confirmée dans son rôle de chef-lieu d'un vaste territoire, quelles que soient les circonstances favorables offertes par le changement de régime du Rhône amorcé depuis le $\mathrm{II}^{\mathrm{e}} \mathrm{s}$., il fallut aussi un programme d'urbanisme ambitieux pour bâtir en cinquante ans 60 ha dans une plaine.

À la période augustéenne, on se préoccupa d'abord de l'organisation du centre monumental qui occupait la majeure partie du promontoire originel. Le forum est établi sur le premier axe de circulation, où il occupe une position symbolique. La construction de ce monument perturbait le fonctionnement traditionnel de l'agglomération en interrompant l'axe carrossable qui la traversait : les marches visibles au pied des arcades du forum en apportent la preuve. L'établissement d'une voie au pied du promontoire, au bord du Rhône, devenait donc vitale pour le commerce et c'est bien à l'époque d'Auguste qu'elle est établie, remplaçant ainsi un simple chemin de berge. Le développement de la ville est particulièrement évident entre l'époque de Tibère et celle de Claude, l'accession au statut de colonie romaine à l'époque de Caligula, voire au début du règne de Claude, donnant certainement encore plus d'ampleur à ce mouvement (Christol, 1994), mais on ne peut croire que le plan d'urbanisme n'ait pas été prévu dès le tournant de notre ère. La stabilité du cours du Rhône, sensible à Vienne dès l'extrême fin du I $^{\text {cr }}$ s. avant J.-C., prouve qu'il était possible d'envisager un tel programme à l'époque d'Auguste. De nouveaux quartiers sont établis sur des dépôts de débordement qui datent au plus tard du tout début du principat. L'exhaussement des terrains réalisé au cours de la première moitié $d u I^{c r} s$. après J.-C. met pour quelques siècles ces quartiers hors de portée des crues. Cet exhaussement pourrait être une réponse à un regain d'activité temporaire du Rhône dont on a des témoins au travers des dépôts de débordement, au sud de la ville, datables des années 30 et qui n'ont pas dépassé la cote $149 \mathrm{~m}$ NGF (fig. $28, \mathrm{n}^{\text {os }} 12,13$, 22). Les zones méridionales, moyennant quelques remblais, furent ensuite partiellement occupées entre le $\mathrm{II}^{\mathrm{c}} \mathrm{s}$. et le début du IV ${ }^{\mathrm{e}}$ s. après J.-C. (fig. $28, \mathrm{n}^{\text {os }} 12,13$ ) : c'est là sans doute la preuve d'une nouvelle longue accalmie. Au-delà de cette période, en revanche, une péjoration très importante du régime fluvial est manifeste. Par exemple, les niveaux d'abandon du quartier romain établi sur d'épais remblais, situé entre la voie Médiane et le Rhône et les nécropoles de la seconde moitié du III's. et du début du $\mathrm{IV}^{\mathrm{c}} \mathrm{s}$., qui se sont installées sporadiquement dans ces ruines, ont été touchés par les crues (fig. 28, $\mathrm{n}^{\circ}$ 22). Peutêtre est-ce à partir du milieu du Ve $\mathrm{V}^{\mathrm{s}}$. qu'il faut dater le début de cette nouvelle péjoration si l'on en croit Grégoire de Tours ( $M G H$, srm, I, p. 564) qui raconte comment saint Mamert, avant 475, dut faire reconstruire, au nord de la plaine de Saint-Romain-en-Gal ( $\vec{a})$ (Reynaud, 1995), la première basilique du saint martyr Ferréol qui avait été bâtie " au-dessus de la rive du Rhône » et qui avait été en partie ruinée « sous les coups du fleuve impétueux». 